\title{
THYROID FUNCTION ASSAY WITH RADIOIODINE. III. CLINI- CAL TEST RESULTS AND DIAGNOSTIC LIMITS FOR RATE CONSTANTS ${ }^{1}$
}

\author{
By T. H. ODDIE,2 I. MESCHAN, AND JAMES T. WORTHAM \\ (From the Department of Radiology and the Department of Medicine, University of Arkansas, \\ Little Rock, Ark.)
}

(Submitted for publication January 24, 1955 ; accepted March 23, 1955)

In an effort to increase the accuracy of assay of thyroidal function, two earlier papers $(1,2)$ have given a numerical and mathematical description of the short-time behavior of radioiodine observed in the neck tissue, thyroid, plasma, and urine. It remains : a) to apply these data to the conversion of several of the diagnostic parameters currently in use in this type of investigation so as to achieve a common basis for comparison; b) to describe the authors' clinical results; and c) to provide a set of diagnostic limits for routine use based upon a maximum amount of published information.

\section{CHOICE OF PARAMETERS}

The various parameters that are commonly employed in the assay of thyroid function with radioiodine are:

(a) The thyroidal plasma clearance rate (3);

(b) The thyroidal fractional rate of uptake from the iodide pool (1);

(c) The 24-hour percentage accumulation in the thyroid gland;

(d) The 24-hour percentage excreted in the urine;

(e) Parameters based on various other bulk samples of urine (4);

(f) The thyroidal rate of output of organic iodine (5); and

(g) The "conversion ratio" (6) of plasma organic and inorganic radioiodine.

Because the mathematical treatment (1) has been restricted to the early phase of iodine uptake, the resulting equations cannot be applied to the

1 Assisted by grants from the National Institute of Health Division of Research Grants, and from the Arkansas Heart Association.

2 Visiting Assistant Professor, Department of Radiology, University of Arkansas, on a Fulbright Fellowship. diagnostic parameters (f) and (g) above, because these are derived from the later (hormone and organic iodine) phases.

In comparing the remaining parameters it is necessary to convert them to some common basis, which is conveniently chosen as either the pair of plasma clearance rates-thyroidal (expressed as g ml. per min.) and renal ( $\mathrm{r} \mathrm{ml}$. per min.) -or the corresponding pair of rate factors-thyroidal $\left(k_{1} \min ^{-1}\right)$ and renal $\left(k_{2} \min ^{-1}\right)$-as defined in an earlier paper (1).

It may be recalled that the clearance rates are equal to the corresponding rate factors multiplied by the volume of the iodide space.

Clearance rates may have slightly more fundamental significance than the rate factors, but in practice their measurement is performed in exactly the same manner except for the additional determination of the radioiodine in a sample of blood plasma; unless the latter is done very carefully indeed, this may add further experimental error to the measured clearance rate while little is gained in diagnostic precision.

At first sight it might seem that a simpler basis of comparison would be provided by the per cent uptake (or excretion) at some selected time (usually 24 hours) after the administration of the test dose, because this is one single parameter rather than a pair. It must be remembered, however, that the per cent uptake really involves both clearance rates (or both rate factors), because a high thyroidal uptake can result either from a high thyroidal rate combined with a normal renal rate or from a moderate thyroidal rate with a low renal rate. Moreover, the possible excretion of organic radioiodine from the thyroid gland may lower the observed per cent uptake to such a value that a hyperthyroid case may appear in the normal range of values; this makes the per cent uptake a one-sided parameter. The per cent excretion 
also has this disadvantage, and it is of course an even more indirect approach to the problem.

In the next section the above parameters are compared on a numerical basis, using data published by others, for those papers where sufficient information has been provided to allow comparison. The search for information of this type has been fairly extensive but not exhaustive, and some papers may have been unintentionally overlooked. There is, however, a whole group of studies, based on the method of Stanley and Astwood (7), that cannot be adapted because these writers have expressed their results in terms of the square root of the time-a mathematical procedure that cannot be linked up with any of the proposed schemes of iodine metabolism. With this group there is the added difficulty that the published numerical parameters depend largely on the different experimental arrangements employed at various centers.

\section{DIAGNOSTIC LIMITS DEDUCED FROM PUBLISHED WORK}

\section{Clearance rates}

From equations $I(4), 3 I(8)$, and $I(9)$ of an earlier paper (1), it is seen that the clearance rates are related to the rate factors by the following :

$$
\begin{aligned}
& \mathrm{k}_{1}=\mathrm{g} / \mathrm{3} \nabla_{0} \\
& \mathrm{k}_{2}=\mathrm{r} / \nabla_{30}
\end{aligned}
$$

8 In this paper equations prefixed $I$ refer to those given in the first paper (1) in this series.
Additional knowledge is needed of the mean reciprocal volume $\left(1 \bar{N}_{30} \mathrm{ml}^{-1}\right)$ of the iodide space for the average patient.

From the information discussed in the same paper (1) the over-all mean value

$$
\mathrm{W} / \mathrm{V}_{30}=3.25 \mathrm{~kg} \cdot / \text { litre }
$$

and also

$$
\nabla_{30}=V_{30} / 1.32
$$

giving, for an average body-weight $\mathrm{W}$ of $70 \mathrm{~kg}$.,

$$
\nabla_{30}=\frac{70,000}{3.25 \times 1.32}=16,300 \mathrm{ml} .
$$

Putting this value in equations (1) and (2) enables the conversion to $k_{1}$ and $k_{2}$ of various published figures for clearance rates, as set out in Table I. Although Berkson, Keating, Power, and McConahey $(8,9)$ deduced their results indirectly from measurements of the radioiodine excreted in the urine, the mid-point of their overlapping values of $\mathbf{g}$, (the thyroidal clearance rate) with normal and hyperthyroid cases, is in good agreement with that of Myant, Corbett, Honour, and Pochin (3); the wide limits of Ansell, Macgregor, Miller, and Wayne (5) are also consistent with these two. On the above results it is apparent that $\mathrm{g}=60 \mathrm{ml}$. per minute or $10^{3} \mathrm{k}_{1}=3.7$ minute $^{-1}$ is the approximate borderline between euthyroid and hyperthyroid cases with normal renal function.

\begin{tabular}{|c|c|c|c|c|c|c|c|}
\hline \multirow[b]{2}{*}{ Authors } & \multirow[b]{2}{*}{$\begin{array}{l}\text { Reference } \\
\text { number }\end{array}$} & \multirow[b]{2}{*}{$\begin{array}{l}\text { Number } \\
\text { of cases }\end{array}$} & \multirow[b]{2}{*}{ Diagnostic category } & \multicolumn{2}{|c|}{$\begin{array}{c}\text { Clearance rate } \\
m \mathrm{ml} . / \mathrm{min} .\end{array}$} & \multicolumn{2}{|c|}{$\begin{array}{c}\text { Deduced rate factors } \\
\text { minute }^{-1}\end{array}$} \\
\hline & & & & Tho. & mose. & $10 k_{1}$ & $\begin{array}{c}10^{3} \mathbf{k}_{2} \\
\text { (mean) }\end{array}$ \\
\hline Myant et al. & 3 & $\begin{array}{l}12 \text { euthyroid } \\
12 \text { Graves' disease }\end{array}$ & $\begin{array}{l}\text { Mid-point of over- } \\
\text { lapping values } \\
\text { normal/hyperthyroid }\end{array}$ & 63 & 30 & 3.87 & 1.84 \\
\hline McConahey et al. & 9 & $\begin{array}{l}9 \text { euthyroid } \\
16 \text { hyperthyroid }\end{array}$ & $\begin{array}{l}\text { Mid-point of over- } \\
\text { lapping values } \\
\text { normal/hyperthyroid }\end{array}$ & 57 & 33 & 3.50 & 2.02 \\
\hline Ansell et al. & 5 & Not stated & $\begin{array}{l}\text { Certainly normal } \\
\text { Upper limit of normal }\end{array}$ & $\begin{array}{l}40 \\
80\end{array}$ & & $\begin{array}{l}2.46 \\
4.52\end{array}$ & \\
\hline Perry and Hughes & 10 & 11 & $\begin{array}{l}\text { Mean value of normal } \\
\text { thyroid but with renal } \\
\text { disease }\end{array}$ & $\begin{array}{r}4.3 \\
\pm 1.5\end{array}$ & $\begin{array}{r}5.5 \\
\pm 1.2\end{array}$ & $\begin{array}{r}0.26 \\
\pm 0.09\end{array}$ & $\begin{array}{r}0.34 \\
\pm 0.07\end{array}$ \\
\hline
\end{tabular}

Perry and Hughes (10) have shown that, when renal function is impaired, the average normal

TABLE I

Conversion of published information on clearance rates 
thyroid values of $g$ (and $k_{1}$ ) are lowered as are the renal parameters; however the number of patients studied is too small to permit a diagnostic boundary value to be set for this type of patient.

\section{Gross 24-hour uptake}

The total neck-tissue-plus-thyroid uptake at 24 hours $(11,12)$ has been a popular parameter at many centers, and optimistic claims have been made for its diagnostic accuracy, presumably because highly hyperthyroid individuals have been tested and compared with definitely normal cases.

To deduce information on $k_{1}$ and $k_{2}$ from the 24-hour uptake one must consider equation $I(11)$

$$
\mathrm{N}=\frac{\mathrm{N}_{0} \mathrm{~V}_{\mathrm{n}}}{\mathrm{V}} \mathrm{e}^{-\mathrm{k} \phi \mathrm{t}}+\left(1-\mathrm{e}^{-\mathrm{k \phi t}}\right) \mathrm{k}_{1} / \mathrm{k} \quad I(11)
$$

which gives the gross uptake provided no radioiodine has left the thyroid gland in organic form; in other words this equation really gives an upper limit for the gross uptake with given values of $k_{1}$ and $k_{2}$. Then if $X$ is the diagnostic boundary value for the 24-hour uptake, higher values being taken as hyperthyroid and lower values as euthyroid one can say that if the right hand side of equation $I(11)$ is not greater than $\mathrm{X}$, then the gross uptake $\mathrm{N}$ also cannot be greater than $\mathrm{X}$ so the case would be a normal one. This may be written as an inequality

$$
\mathrm{N} \leq \frac{\mathrm{N}_{0} \mathrm{~V}_{\mathrm{n}}}{\mathrm{V}} \mathrm{e}^{-\mathrm{k} \phi t}+\left(1-\mathrm{e}^{-\mathrm{k \phi t}}\right) \mathrm{k}_{1} / \mathrm{k} \leq \mathrm{X}
$$

On the other hand if the formula leads to a quantity greater than $\mathrm{X}$ one cannot be sure whether $\mathrm{N}$ is greater or less than $\mathrm{X}$ so the diagnosis is in doubt. Thus writing

$$
\frac{N_{0} V_{n}}{V} e^{-k \phi t}+\left(1-e^{-k \phi t}\right) k_{1} / k=X
$$

leads to a value of $k_{1}$ for any given value of $k_{2}$, because $k=k_{1}+k_{2}$. Then if $k_{1}$ for any patient is less than this value the patient must be euthyroid. Still there will be a region of doubtful $\mathbf{k}_{\mathbf{1}}$ values just above this boundary. This explains one of the major disadvantages of the 24-hour parameter; another, less critical, difficulty is the uncertainty in the term $\mathrm{N}_{0} \mathrm{~V}_{\mathrm{n}} / \mathrm{V}$ unless it has been determined by experiment for the exact geometrical arrangement used for in vivo counting.

In an early paper Werner, Quimby, and Schmidt (12) set a boundary value of 35 per cent $(X=0.35)$ for the 24-hour uptake to distinguish between euthyroid and hyperthyroid cases. Jaffe and Ottoman (11) published a similar value, and this appears to have been adopted quite widely by other centers, at least in this country. Recently, however, Werner (13) has used a much higher borderline of 50 to 55 per cent, which is in better agreement with the value to be expected when calculated from the other parameters. The ranges of uptakes given by Fields and LeRoy (14) are also more consistent with the higher boundary.

Taking as an example Werner's limit of $\mathrm{X}=$ 0.55 , and $\mathrm{N}_{\mathrm{o}} \mathrm{V}_{\mathrm{n}} / \mathrm{V}=0.063$ at $\mathrm{t}=24$ hours-an estimate made from comparative observations on an athyroid patient using a Geiger counter arranged in the manner described by these authors (12) - equation (5) becomes, with $\phi \mathrm{t}=566 \mathrm{~min}$ utes,

$$
.063 \mathrm{e}^{-566 \mathrm{k}}+\left(1-\mathrm{e}^{-566 \mathrm{k}}\right) \mathrm{k}_{1} / \mathrm{k}=0.55
$$

Pairs of $k$ values can be found to satisfy (6), typical cases being included in Table III and in Figure 1.

\section{Urinary excretion}

Methods for studying thyroid activity by means

\begin{tabular}{|c|c|c|c|c|}
\hline \multirow[b]{2}{*}{ Type of case } & \multirow{2}{*}{$\begin{array}{l}\text { Number } \\
\text { of cases }\end{array}$} & \multicolumn{2}{|c|}{$\begin{array}{c}\text { Average rate factors } \\
\text { deduced from } \\
\text { published data } \\
\text { minute }^{-1}\end{array}$} & \multirow{2}{*}{$\begin{array}{c}\text { Range* } \\
\text { for } \\
\text { uptake } \\
\text { rate } \\
10^{3} k_{1} \\
\text { minute-1 }\end{array}$} \\
\hline & & $\log _{k_{1}}$ & $10 \mathrm{k}_{2}$ & \\
\hline $\begin{array}{l}\text { Normal } \\
\text { (controls) }\end{array}$ & 4 & 1.8 & 3.4 & $0.5-3.2$ \\
\hline $\begin{array}{l}\text { Euthyroid } \\
\text { (but unrelated } \\
\text { illness) } \\
\text { Non-toxic goiter } \\
\text { Toxic goiter } \\
\text { Exophthalmic }\end{array}$ & $\begin{array}{l}28 \\
26 \\
23\end{array}$ & $\begin{array}{l}1.8 \\
2.7 \\
3.7\end{array}$ & $\begin{array}{l}3.0 \\
3.4 \\
2.3\end{array}$ & $\begin{array}{l}1.3-2.4 \\
1.6-3.7 \\
0.7-6.7\end{array}$ \\
\hline $\begin{array}{l}\text { goiter } \\
\text { Myxedema }\end{array}$ & $\begin{array}{l}64 \\
24\end{array}$ & $\begin{array}{l}9.1 \\
0.5\end{array}$ & $\begin{array}{l}2.0 \\
2.8\end{array}$ & $\begin{array}{r}7.7-10.5 \\
0-0.6\end{array}$ \\
\hline
\end{tabular}
of the urinary excretion of radioiodine fall into

TABLE II

Conversion of data reported by Keating and his associates

* The range tabulated here is the mean value \pm twice the standard deviation given in the original paper $(15,16)$. 
TABLE III

Conversion of Werner's (13) 24-hour uptake limit and Fraser and others (4) urinary T-parameter limits

\begin{tabular}{|c|c|c|c|c|c|}
\hline \multirow[b]{2}{*}{$\begin{array}{c}\text { Renal rate } \\
10^{0} k_{2} \\
\text { minute-1 }\end{array}$} & \multicolumn{5}{|c|}{$\begin{array}{l}\text { Deduced values of the thyroid rate } \\
\qquad 0^{0} \mathrm{k}_{1} \text { minute }^{-1}\end{array}$} \\
\hline & $\begin{array}{c}T=2.1 \\
\text { Very low/ } \\
\text { low uptake } \\
\text { borderline }\end{array}$ & $\begin{array}{c}T=2.8 \\
\text { Low/normal } \\
\text { uptake } \\
\text { borderline }\end{array}$ & $\begin{array}{c}\mathrm{T}=12.8 \\
\text { Normal/ } \\
\text { probably } \\
\text { high } \\
\text { borderline }\end{array}$ & $\begin{array}{l}\text { 24-hour } \\
\text { gross uptake } \\
55 \text { per cent } \\
\text { Werner } \\
\text { upper limit } \\
\text { of normal }\end{array}$ & $\begin{array}{c}\text { T }=17.4 \\
\text { Probably } \\
\text { high/ } \\
\text { high } \\
\text { borderline }\end{array}$ \\
\hline $\begin{array}{l}0.1 \\
0.2 \\
0.3 \\
0.4 \\
0.7 \\
1 \\
2 \\
3 \\
4\end{array} \quad \begin{array}{c}\text { Normal renal } \\
\text { range }\end{array}$ & $\begin{array}{l}- \\
- \\
\bar{Z} \\
0.17 \\
0.40 \\
0.53 \\
0.20 \\
-\end{array}$ & $\begin{array}{l}- \\
- \\
0.09 \\
0.57 \\
0.85 \\
1.04 \\
0.81 \\
0.22\end{array}$ & $\begin{array}{l}0.50 \\
1.50 \\
2.08 \\
2.46 \\
3.21 \\
3.63 \\
4.22 \\
4.18 \\
3.95\end{array}$ & $\begin{array}{l}1.34 \\
1.41 \\
1.47 \\
1.53 \\
1.74 \\
1.97 \\
2.81 \\
3.80 \\
4.89\end{array}$ & $\begin{array}{l}0.92 \\
1.98 \\
2.57 \\
3.03 \\
3.82 \\
4.32 \\
4.95 \\
4.98 \\
4.70\end{array}$ \\
\hline
\end{tabular}

two main classes, (a) the analytical method with simple exponential excretion curves which has been used extensively by Keating and his associates $(15,16)$, and (b) the bulk sample methods of which a recent example is that described by Fraser, Hobson, Arnott, and Emery (4).

Keating's method assumes a simple exponential relation, equivalent to putting $\phi=1$ in the pres-

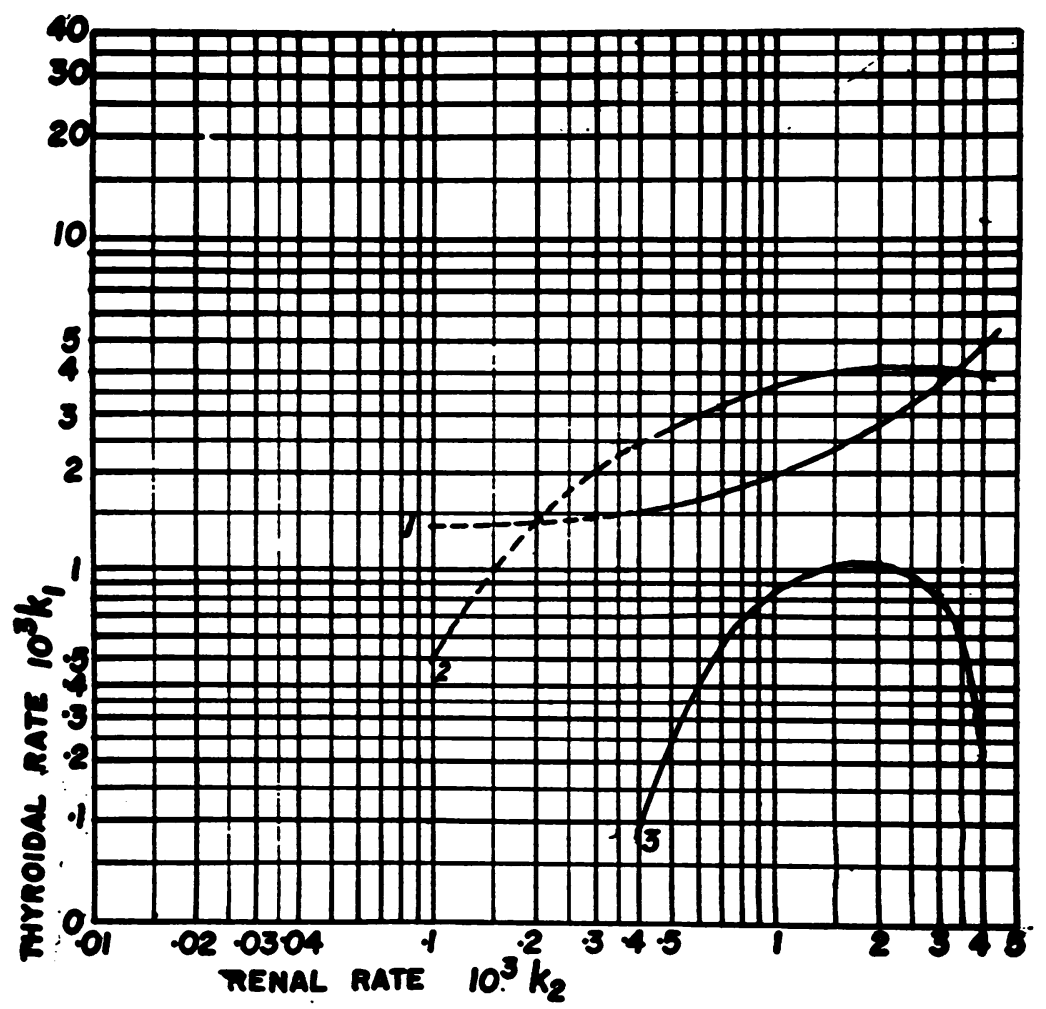

Fig. 1. Typical Diagnostic Borderlines Deduced for 24-Hour Uptake and Bulk Urine Methods Using Radioiodine

Curve 1-24-hour uptake 55 per cent, reference (13).

Curve 2-Bulk urine method, euthyroid/probably high borderline $T=12.8$, reference (4).

Curve 3-As above, low/euthyroid borderline $T=2.8$. 
ent authors' equations. With this assumption equation $I(7)$ may be written

$$
\mathrm{kt}=-\ln \left(1-\frac{\mathrm{kE}}{\mathrm{k}_{2}}\right)
$$

and, by plotting the observed results for the urine fraction $E$ against the time $t$ in this form on semilogarithmic paper, a straight line should result. Actually since $\phi$ does not vary very rapidly with $t$ after the first hour the more precise relation $I(7)$ also approximates to a straight line over limited periods of time, and moderate departures from linearity are easily masked by experimental errors in the individual determinations of $\mathrm{E}$. A further masking of these departures can result from the oral rather than the intravenous administration of the test dose, causing "zero" time to be spread out over a period of some minutes.

If equations $I(7)$ and (7) are compared it is apparent that the effective average slope of the experimental curve between two times $t_{1}$ and $t_{2}$ will be given approximately by

$$
k_{\text {effoctive }}=k\left(\frac{\phi_{2} t_{2}-\phi_{1} t_{1}}{t_{2}-t_{1}}\right)
$$

Putting in values (1) of $\phi$ gives the following results :

$$
\begin{gathered}
\begin{array}{c}
\text { Period of } \\
\text { observation } \\
\text { in hours }
\end{array} \\
1 \text { to } 10 \\
3 \text { to } 24 \\
3 \text { to } 72
\end{gathered}
$$

$$
\begin{gathered}
\begin{array}{c}
\text { Approximate } \\
\text { average } \\
\text { rate koffoetive }
\end{array} \\
0.45 \mathrm{k} \\
0.36 \mathrm{k} \\
0.32 \mathrm{k}
\end{gathered}
$$

The period up to 10 hours covers all the early useful range of urine measurements that can be made on a hyperthyroid patient, while measurements on hypothyroid cases can be extended up to 72 hours or more. Thus the conversion of effective (Keating-type) rates to $\mathbf{k}_{\mathbf{1}}$ or $\mathbf{k}_{\mathbf{2}}$ values depends on the type of case being considered, but arbitrarily taking the three factors tabulated above and applying them to some of the published results (16) for (respectively) hyperthyroid, euthyroid, and hypothyroid cases, lead to $k_{1}$ and $k_{2}$ values as shown in Table II. The ranges of $k_{1}$ show a considerable overlap for the euthyroid and hyperthyroid groups, possibly caused by breakdown of organically-bound radioiodine during the course of the test in some instances. From the figures tabulated it is difficult to pick a diagnostic boundary with any confidence, but the euthyroid/hyperthyroid border would appear to be somewhere about $10^{3} \mathrm{k}_{1}=3.7$ to 4.2 minute $^{-1}$, and the euthyroid/hypothyroid border about $10^{3} \mathrm{k}_{1}$ $=0.5$ minute $^{-1}$. The overall average value of the renal rate is $10^{3} \mathrm{k}_{2}=2.6$ minute $^{-1}$.

The urinary parameter $\mathrm{T}$ taken by Fraser, Hobson, Arnott, and Emery (4) may be represented by

$$
\mathrm{T}=\frac{\text { Fraction of dose excreted up to } 8 \text { hours }}{\text { (Fraction excreted from } 8 \text { to } 24 \text { hours) }}
$$

which, in the present representation, becomes, with $\phi$ values inserted (1),

$$
\mathrm{T}=\frac{1-\mathrm{e}^{-247 \mathrm{k}}}{\left(\mathrm{e}^{-247 \mathrm{k}}-\mathrm{e}^{-566 \mathrm{k}}\right)\left(1-\mathrm{e}^{-1003 \mathrm{k}}\right) \mathrm{k}_{2} / \mathrm{k}}
$$

Boundary values of $T$ in the original paper (17) are :

High uptake- $\mathrm{T}$ greater than 17.4

Probably high-T greater than 12.8 , up to 17.4

Normal-T from 2.8 to 12.8

Low-T from 2.1, but less than 2.8

Very low-T less than 2.1

These values may be produced by putting into equation (9) the pairs of $k_{1}$ and $k_{2}$ values shown in Table III, but of course there is some uncertainty about the exact range of $k_{2}$ values over which the $T$ parameter may be expected to be valid. Lacking information on this point it is assumed that this range would extend, for cases without renal or cardiac disease, from $10^{3} \mathrm{k}_{2}=0.4$ to 4.2 minute $^{-1}$ approximately. The curves corresponding to the parameters $\mathrm{T}=2.8$ and $\mathrm{T}=$ 12.8 are shown for comparison in Figure 1.

\section{Early uptake methods}

The remaining type of information to be considered covers those observations made of the thyroidal uptake within the first few hours after administering the radioiodine.

In this category are several papers with data that are difficult to convert to the required numerical basis for comparison: thus the results of Reiss, Haigh, Hemphill, Maggs, Reiss, and Smith (17) have been excluded for lack of sufficiently accurate knowledge of their observed neck tissue uptake and other factors; the uptakes of Lowrey, Beierwalters, Lampe, and Gomberg (18) in a 
TABLE IV

Results obtained on patients tested by protein-bound iodine and radioiodine methods

\begin{tabular}{|c|c|c|c|c|c|c|c|}
\hline 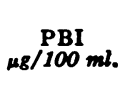 & $\begin{array}{l}\text { Rate factor } \\
10^{3} \mathbf{k}_{1} \\
\min .^{-1}\end{array}$ & $\begin{array}{c}\text { Combined } \\
\text { parameter } \\
z\end{array}$ & $\begin{array}{c}\text { 24-Hour } \\
\text { uptake } \\
\%\end{array}$ & $\underset{\mu \mathrm{PBI} / 100 \mathrm{ml} .}{\text { PBI }}$ & $\begin{array}{c}\text { Rate factor } \\
10^{4} k_{1} \\
\min ^{-1}\end{array}$ & $\begin{array}{l}\text { Combined } \\
\text { parameter } \\
z\end{array}$ & $\begin{array}{c}\text { 24-Hour } \\
\text { uptake } \\
\%\end{array}$ \\
\hline \multicolumn{8}{|c|}{ Cases classed as hypothyroid } \\
\hline $\begin{array}{l}0.3 \\
0.5 \\
0.6 \\
0.6 \\
0.7\end{array}$ & $\begin{array}{l}0 \\
0 \\
0 \\
0.02\end{array}$ & & $\begin{array}{l}3.1 \\
0.8 \\
3.0\end{array}$ & $\begin{array}{l}3.3 \\
3.5 \\
3.5 \\
3.6 \\
3.6\end{array}$ & $\begin{array}{l}0 \\
0 \\
0.10 \\
0\end{array}$ & & 17.4 \\
\hline $\begin{array}{l}0.9 \\
1.0 \\
1.0 \\
1.1 \\
1.2\end{array}$ & $\begin{array}{l}0 \\
0 \\
0 \\
0 \\
0.17\end{array}$ & & $\begin{array}{r}0.4 \\
1.7 \\
2.6 \\
14.3 \\
8.2\end{array}$ & $\begin{array}{l}3.8 \\
3.8 \\
3.9 \\
4.4 \\
4.5\end{array}$ & $\begin{array}{l}0 \\
0.21 \\
0.18 \\
0 \\
0.16\end{array}$ & $\begin{array}{l}2.10 \\
3.66\end{array}$ & $\begin{array}{r}15.4 \\
6.1\end{array}$ \\
\hline $\begin{array}{l}1.8 \\
2.0 \\
2.1 \\
2.5 \\
2.7\end{array}$ & $\begin{array}{l}0.10 \\
0.03 \\
0.23 \\
0.19 \\
0.28\end{array}$ & & $\begin{array}{r}1.2 \\
17.6\end{array}$ & $\begin{array}{l}5.2 \\
5.5 \\
5.8 \\
6.6\end{array}$ & $\begin{array}{l}0.06 \\
0.08\end{array}$ & $\begin{array}{l}3.28 \\
3.56\end{array}$ & $\begin{array}{r}3.9 \\
12.5 \\
3.3\end{array}$ \\
\hline $\begin{array}{l}2.7 \\
2.9 \\
2.9 \\
3.0 \\
3.2\end{array}$ & $\begin{array}{l}0 \\
0.10 \\
0.18 \\
0.16\end{array}$ & & $\begin{array}{r}4.8 \\
1.0 \\
15.2 \\
15.0\end{array}$ & & $\begin{array}{l}0 \\
0 \\
0 \\
0.05 \\
0.11\end{array}$ & & $\begin{array}{l}1.2 \\
1.7 \\
4.0 \\
2.0 \\
7.7\end{array}$ \\
\hline \multicolumn{8}{|c|}{ Cases classed as euthyroid } \\
\hline $\begin{array}{l}2.5 \\
2.8 \\
3.0 \\
3.2 \\
3.2\end{array}$ & $\begin{array}{l}1.15 \\
2.30 \\
1.71 \\
0.80 \\
0.75\end{array}$ & $\begin{array}{l}4.22 \\
4.70 \\
5.86 \\
4.23 \\
4.19\end{array}$ & $\begin{array}{l}31.2 \\
26.0\end{array}$ & $\begin{array}{l}4.5 \\
4.6 \\
4.6 \\
4.6 \\
4.6\end{array}$ & $\begin{array}{l}0.81 \\
0.65 \\
0.21 \\
0.65 \\
0.25\end{array}$ & 3.84 & $\begin{array}{l}32.0 \\
22.9 \\
15.9 \\
20.0 \\
44.2\end{array}$ \\
\hline $\begin{array}{l}3.2 \\
3.2 \\
3.4 \\
3.8 \\
3.9\end{array}$ & $\begin{array}{l}0.40 \\
0.65 \\
1.86 \\
0.43\end{array}$ & $\begin{array}{l}3.84 \\
4.17 \\
4.07\end{array}$ & $\begin{array}{l}19.0 \\
23.6 \\
33.5 \\
43.3\end{array}$ & $\begin{array}{l}4.6 \\
4.6 \\
4.7 \\
4.7 \\
4.7\end{array}$ & $\begin{array}{l}0.31 \\
1.90 \\
0.28 \\
0.74 \\
0.34\end{array}$ & & $\begin{array}{l}17.4 \\
54.0 \\
17.5 \\
41.4\end{array}$ \\
\hline $\begin{array}{l}4.0 \\
4.0 \\
4.0 \\
4.1 \\
4.1\end{array}$ & $\begin{array}{l}0.24 \\
0.58 \\
0.52 \\
1.18 \\
0.52\end{array}$ & & 13.4 & $\begin{array}{l}4.7 \\
4.7 \\
4.8 \\
4.8 \\
4.9\end{array}$ & $\begin{array}{l}0.71 \\
1.81\end{array}$ & & $\begin{array}{l}30.7 \\
12.7 \\
40.6 \\
21.9\end{array}$ \\
\hline $\begin{array}{l}4.2 \\
4.2 \\
4.2 \\
4.2 \\
4.2\end{array}$ & $\begin{array}{l}0.22 \\
0.75 \\
1.28 \\
0.33 \\
0.55\end{array}$ & 3.77 & 18.0 & $\begin{array}{l}5.0 \\
5.0 \\
5.0 \\
5.0 \\
5.0\end{array}$ & $\begin{array}{l}1.56 \\
0.25 \\
1.83 \\
0.32 \\
0.49\end{array}$ & & $\begin{array}{l}17.0 \\
10.7 \\
24.1\end{array}$ \\
\hline $\begin{array}{l}4.3 \\
4.3 \\
4.4 \\
4.4 \\
4.5\end{array}$ & $\begin{array}{l}0.62 \\
0.29 \\
1.10 \\
0.75 \\
0.29\end{array}$ & & $\begin{array}{l}15.8 \\
42.3 \\
25.7\end{array}$ & $\begin{array}{l}5.1 \\
5.1 \\
5.2 \\
5.2 \\
5.2\end{array}$ & $\begin{array}{l}0.17 \\
0.45 \\
1.40 \\
1.20 \\
1.35\end{array}$ & 3.83 & \\
\hline $\begin{array}{l}4.5 \\
4.5 \\
4.5 \\
4.5 \\
4.5\end{array}$ & $\begin{array}{l}0.27 \\
0.61 \\
0.84 \\
0.67 \\
0.42\end{array}$ & & $\begin{array}{l}12.7 \\
31.4 \\
31.1 \\
14.5\end{array}$ & $\begin{array}{l}5.2 \\
5.2 \\
5.2 \\
5.3 \\
5.3\end{array}$ & $\begin{array}{l}0.32 \\
0.25 \\
0.61\end{array}$ & & $\begin{array}{l}17.4 \\
38.9\end{array}$ \\
\hline
\end{tabular}


TABLE IV-Continued

\begin{tabular}{|c|c|c|c|c|c|c|c|}
\hline$\underset{\mu \mathrm{PBI} / 100 \mathrm{ml} .}{\mathrm{PBI}}$ & 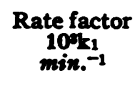 & $\underset{z}{\text { Combinined }}$ & $\begin{array}{c}\text { 24-Hour } \\
\text { uptake } \\
\%\end{array}$ & $\underset{\mu g / 100 \mathrm{ml} .}{\text { PBI }}$ & $\begin{array}{c}\text { Rate factor } \\
10^{3} x_{1} \\
\text { min. }^{-1}\end{array}$ & $\underset{\mathbf{z}}{\text { Combined }}$ & $\begin{array}{c}\text { 24-Hour } \\
\text { uptake } \\
\%\end{array}$ \\
\hline \multicolumn{8}{|c|}{ Cases classed as euthyroid-Continued } \\
\hline $\begin{array}{l}5.3 \\
5.3 \\
5.3 \\
5.4 \\
5.4\end{array}$ & $\begin{array}{l}0.65 \\
0.95 \\
0.28 \\
0.61 \\
0.56\end{array}$ & & 21.1 & $\begin{array}{l}5.9 \\
6.0 \\
6.0 \\
6.0 \\
6.3\end{array}$ & $\begin{array}{l}0.46 \\
0.92 \\
0.66 \\
0.28 \\
1.01\end{array}$ & & 18.7 \\
\hline $\begin{array}{l}5.4 \\
5.5 \\
5.5 \\
5.5 \\
5.5\end{array}$ & $\begin{array}{l}0.91 \\
0.22 \\
0.99 \\
0.55 \\
0.36\end{array}$ & 4.06 & $\begin{array}{r}5.4 \\
43.0 \\
23.8\end{array}$ & $\begin{array}{l}6.4 \\
6.4 \\
6.4 \\
6.5 \\
6.5\end{array}$ & $\begin{array}{l}0.38 \\
0.80 \\
0.65 \\
3.80 \\
0.70\end{array}$ & & $\begin{array}{l}18.4 \\
25.0\end{array}$ \\
\hline $\begin{array}{l}5.5 \\
5.5 \\
5.6 \\
5.6 \\
5.6\end{array}$ & $\begin{array}{l}0.31 \\
0.18 \\
1.16 \\
0.71\end{array}$ & 3.97 & $\begin{array}{r}30.0 \\
7.8 \\
33.9 \\
29.1\end{array}$ & $\begin{array}{l}6.5 \\
6.8 \\
6.9 \\
7.0 \\
7.2\end{array}$ & $\begin{array}{l}0.52 \\
3.30 \\
1.75 \\
0.41\end{array}$ & & $\begin{array}{l}11.1 \\
21.5\end{array}$ \\
\hline $\begin{array}{l}5.6 \\
5.6 \\
5.7 \\
5.7 \\
5.7\end{array}$ & $\begin{array}{l}0.81 \\
0.68 \\
0 \\
2.66 \\
0.59\end{array}$ & 2.39 & $\begin{array}{l}17.5 \\
10.3\end{array}$ & $\begin{array}{l}7.2 \\
7.4 \\
7.4 \\
7.5 \\
7.5\end{array}$ & $\begin{array}{l}0.57 \\
0.61 \\
0\end{array}$ & 2.74 & $\begin{array}{r}7.2 \\
24.2 \\
28.0 \\
22.9\end{array}$ \\
\hline $\begin{array}{l} \\
5.7 \\
5.8 \\
5.8 \\
5.8\end{array}$ & $\begin{array}{l}1.93 \\
0.48 \\
0.69 \\
0.34\end{array}$ & & $\begin{array}{l}41.3 \\
21.0\end{array}$ & $\begin{array}{l}7.6 \\
7.8 \\
7.9 \\
8.0 \\
8.0\end{array}$ & $\begin{array}{l}1.10 \\
0.34 \\
2.08 \\
0.50 \\
1.70\end{array}$ & & $\begin{array}{l}22.4 \\
15.9 \\
32.4 \\
26.2 \\
37.6\end{array}$ \\
\hline 5.8 & 0.43 & & & $\begin{array}{r}8.2 \\
9.8\end{array}$ & $\begin{array}{l}0.24 \\
0.71\end{array}$ & 5.50 & \\
\hline $\begin{array}{l}5.9 \\
5.9 \\
5.9 \\
5.9\end{array}$ & $\begin{array}{l}1.19 \\
0.55 \\
2.40 \\
1.60\end{array}$ & & 31.3 & $\begin{array}{l}10.0 \\
10.1 \\
13.3\end{array}$ & 0.73 & 6.03 & $\begin{array}{l}11.1 \\
14.0 \\
36.9\end{array}$ \\
\hline 5.9 & 3.40 & & 27.7 & & 0.51 & & 26.2 \\
\hline \multicolumn{8}{|c|}{ Cases classed as hyperthyroid } \\
\hline $\begin{array}{l}5.8 \\
5.9 \\
8.2 \\
8.3 \\
9.3\end{array}$ & $\begin{array}{r}6.0 \\
4.1 \\
4.6 \\
15.3 \\
15.8\end{array}$ & & & $\begin{array}{l}10.6 \\
12.0 \\
12.2 \\
13.0 \\
13.6\end{array}$ & $\begin{array}{r}9.8 \\
11.0 \\
9.0 \\
16.0 \\
17.5\end{array}$ & & \\
\hline $\begin{array}{r}9.7 \\
9.8 \\
9.9 \\
10.2 \\
10.4\end{array}$ & $\begin{array}{r}7.0 \\
200 \\
.4 \\
16.0 \\
4.0\end{array}$ & & & $\begin{array}{l}15.4 \\
16.8 \\
17.2 \\
17.7\end{array}$ & $\begin{array}{l}24.0 \\
14.3 \\
34.7 \\
32.6\end{array}$ & & \\
\hline
\end{tabular}

small group of children have been omitted for similar reasons; and Crispell, Parson, and Sprinkle (19) have given too meagre details of their counting arrangement.

Apart from the above, there are the Melbourne, Australia, figures of Clarke and Aujard (20) who give a euthyroid/hyperthyroid borderline value of 4.5 to 5.0 per cent for the increase in thy- roid uptake between 15 and 25 minutes after injection; since they applied a correction for neck tissue counts, based on thigh counts at frequent intervals, one can express this limit in the present terminology as

$$
\left(e^{-17.7 k}-e^{-26.1 k}\right) k_{1} / k=0.0475
$$

using the values of $\phi t$ presented earlier (1) 
TABLE V

Information from early uptake observations

\begin{tabular}{|c|c|c|c|c|c|}
\hline \multirow[b]{2}{*}{ Authors } & \multirow{2}{*}{$\begin{array}{l}\text { Number } \\
\text { of cases }\end{array}$} & \multirow[b]{2}{*}{ Diagnostic category } & \multicolumn{3}{|c|}{ Rate factors in minute -1} \\
\hline & & & $10^{2} k_{1}$ range & $10^{3} k_{2}$ range & $10 x_{2}$ mean \\
\hline $\begin{array}{l}\text { Berson, } \\
\text { Yalow, } \\
\text { Sorrentino, } \\
\text { and Roswit (22) }\end{array}$ & $\begin{array}{r}5 \\
87 \\
18 \\
110\end{array}$ & $\begin{array}{l}\text { Hypothyroid } \\
\text { Euthyroid } \\
\text { Hyperthyroid } \\
\text { All categories }\end{array}$ & $\begin{array}{l}0.00-0.32 \\
0.30-3.51 \\
5.5-38.7\end{array}$ & $\begin{array}{l}0.70-2.95 \\
0.88-4.24 \\
0.42-3.85\end{array}$ & $\begin{array}{l}2.01 \\
2.65 \\
2.42 \\
2.59\end{array}$ \\
\hline $\begin{array}{l}\text { This } \\
\text { paper }\end{array}$ & $\begin{array}{r}14 \\
28 \\
52 \\
75 \\
1 \\
22 \\
67 \\
125\end{array}$ & $\begin{array}{l}\text { Hypothyroid, cardiac } \\
\text { Hypothyroid, non-cardiac } \\
\text { Euthyroid, cardiac } \\
\text { Euthyroid, non-cardiac } \\
\text { Hyperthyroid, cardiac } \\
\text { Hyperthyroid, non-cardiac } \\
\text { All categories, cardiac } \\
\text { All categories, non-cardiac }\end{array}$ & $\begin{array}{c}0-0.21 \\
0-0.28 \\
0-1.83 \\
0.18-3.80 \\
(9.0) \\
4.0-34.7\end{array}$ & $\begin{array}{c}0.04-1.10 \\
0.08-2.30 \\
0.02-3.59 \\
0.09-3.90 \\
(0.73) \\
0.02-4.7\end{array}$ & $\begin{array}{l}0.40 \\
1.21 \\
0.94 \\
1.53 \\
0.73 \\
1.82 \\
0.84 \\
1.51\end{array}$ \\
\hline
\end{tabular}

For $k_{2}$ in the expected range ( 0.4 to $4.2 \times$ $10^{-3}$ minute $\left.^{-1}\right)$ it is easily shown that $k_{1}$ needs to be between 6.6 and $7.2 \times 10^{-3}$ minute $^{-1}$ to satisfy this relation.

By a similar line of reasoning, figures given by Kriss (21) for the net uptake in a few patients one hour after injection lead to a euthyroid/hy- perthyroid borderline of $k_{1}$ ranging from 2.5 to $2.8 \times 10^{-3}$ minute $^{-1}$; in his paper Kriss makes no mention of the application of any corrections for loss of radioiodine left behind in the injection syringe or for absorption and backscatter in the patient, so the accuracy of these figures is in doubt.

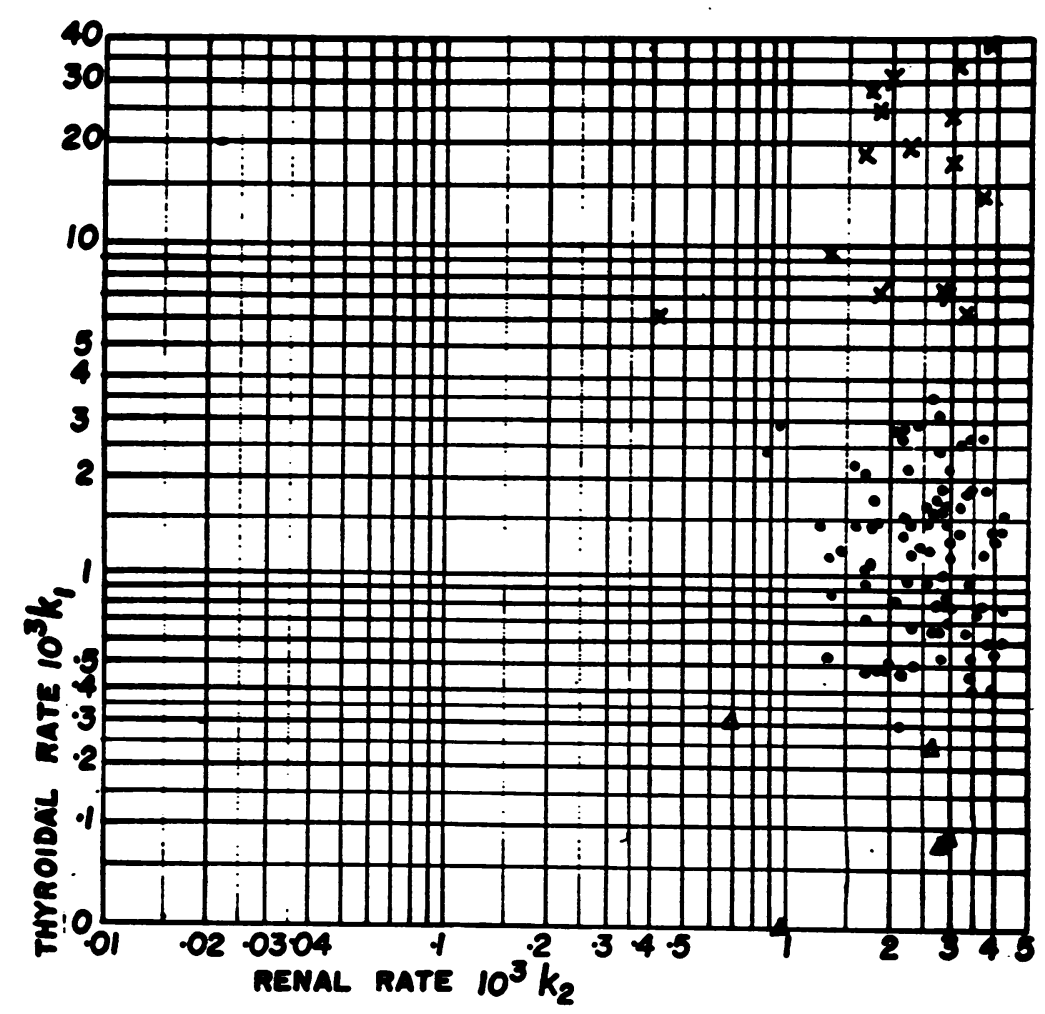

Fig. 2. Expertmental Results of Berson, Yalow, Sorrentino, and Roswit

$\times$ Hyperthyroid $\bullet$ Euthyroid $\triangle$ Hypothyroid 
Finally, test results for the New York area are given in the paper by Berson, Yalow, Sorrentino, and Roswit (22), nominally in the form of clearance rates calculated assuming a constant ratio of $W / \nabla_{30}=5.0 \mathrm{~kg}$./litre; since full data are tabulated these rates are easily converted back to $k_{1}$ and $k_{2}$ values by using equation $I(4)$, with the results given in Table $\mathrm{V}$ and Figure 2.

\section{CLINICAL RESULTS OBTAINED BY THE AUTHORS}

Using the methods previously described $(1,2)$, a number of hospital patients have been examined to relate their thyroidal status to the observed rate factor $k_{1}$; the results are given in Table IV. The status of each patient was decided independently: (i) on clinical grounds; (ii) on the basis of a protein-bound iodine determination, with tentative borderlines of hypothyroid/euthyroid $\mathrm{PBI}=4.0$ micrograms iodine per $100 \mathrm{ml}$. and euthyroid/hyperthyroid $\mathrm{PBI}=8.0$; and (iii) by the radioiodine test with tentative $k_{1}$ limits chosen from the published data discussed above. To decide a few clinically doubtful cases equal weight was given to the $\mathrm{PBI}$ and $\mathrm{I}^{131}$ tests, ex- pressing the combined diagnostic parameter in the form

$$
Z=(P B I)^{1}+1.28 \log _{10}\left(10^{5} k_{1}\right)
$$

with diagnostic values of $Z=3.72$ for the hypothyroid/euthyroid borderline, and $Z=6.17$ for the euthyroid/hyperthyroid boundary.

This type of parameter was adopted because a plot of the mean experimental values showed an approximate linear correlation between the square root of the PBI and the logarithm of the observed $k_{1}$. Figure 3 shows this relation, where each point plotted is the mean of about fifteen observations.

Table $\mathrm{V}$ summarizes the numerical values found for $k_{1}$ and $k_{2}$ for patients in various categories and Figure 4 shows these results in the form of a chart. The low values of the renal rate factor $k_{2}$ included in Figure 4 arise mainly from the inclusion of cardiac cases.

In some of the tests additional information has been obtained in the form of the percentage uptake of $\mathrm{I}^{131}$ at 24 hours after injection, and, in a few cases only, the PBI ${ }^{131}$ "conversion ratio" at 72 hours as described by Silver, Fieber, and Yo-

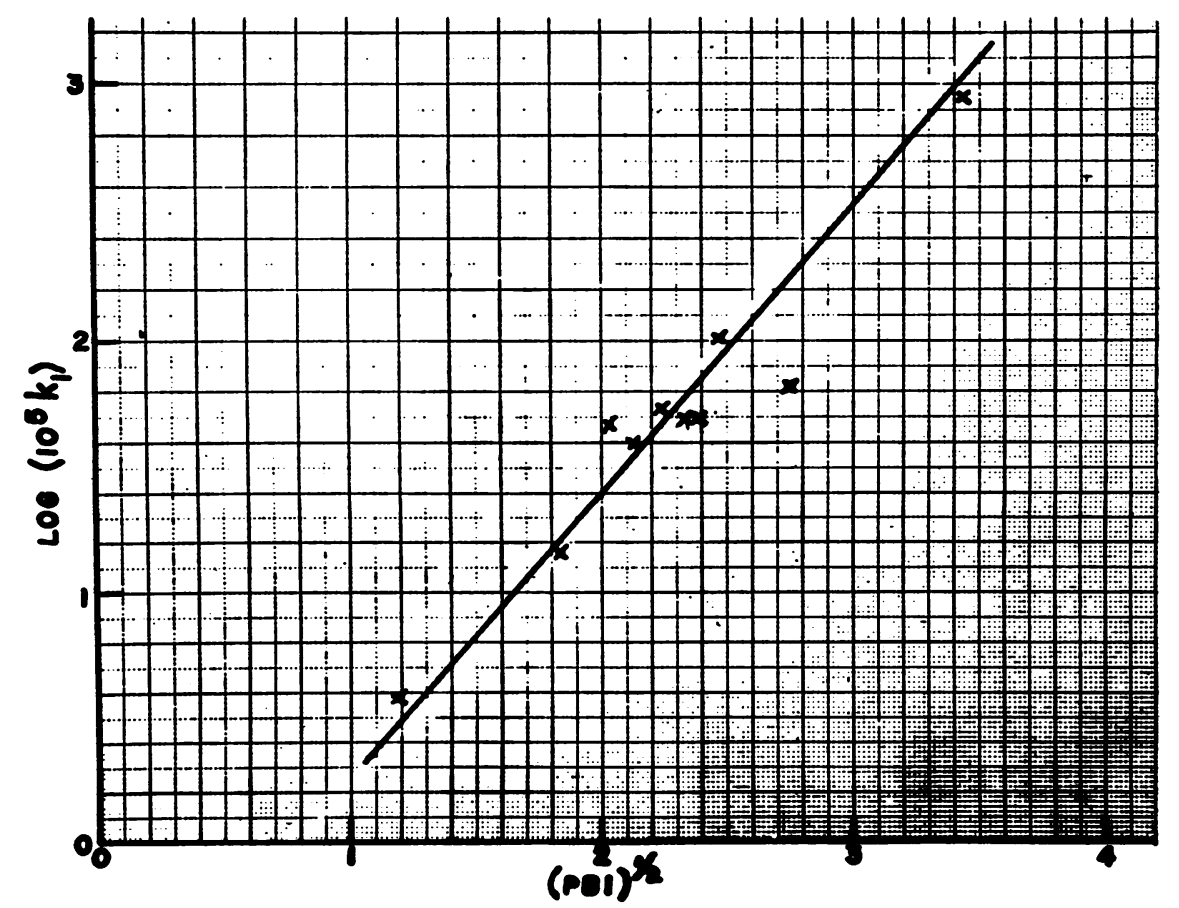

Fig. 3. Mean Exprrimental Points Plotted to Show the Approximate Rriation Between PBI and Rate Factor $K_{1}$ 


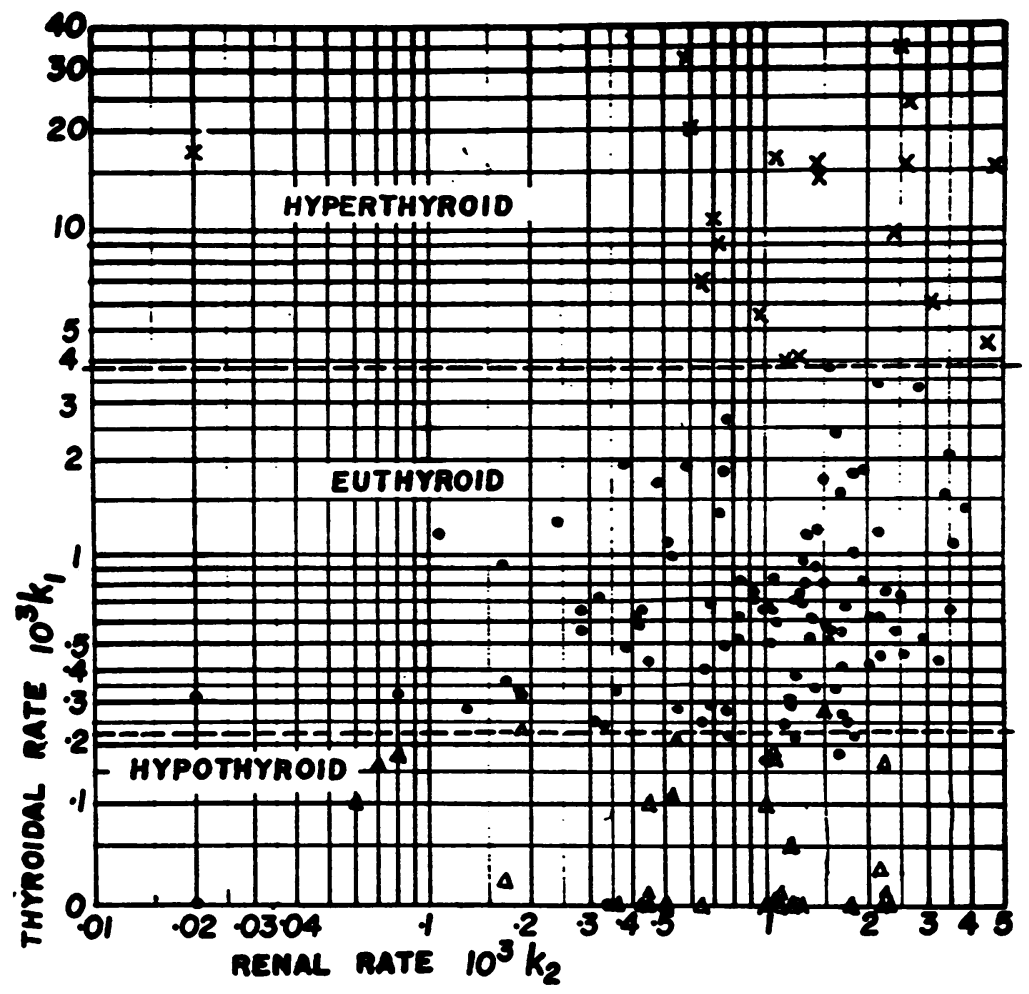

Fig. 4. Experingental Results Obtained by the Authors $\times$ Hyperthyroid $\bullet$ Euthyroid $\triangle$ Hypothyroid

halem (23); the 24-hour uptakes are included in Table IV for purposes of comparison.

\section{DISCUSSION}

\section{Renal rate factor $k_{2}$}

From the experimental data described above there seems to be little evidence that the renal rate $\left(k_{2}\right.$ or $\left.r\right)$ has any significant effect on the thyroidal rate $\left(k_{1}\right.$ or $g$ ) for a given thyroid status; this is shown most clearly by Figures 2 and 4, in which horizontal borderlines, parallel to the $k_{2}$ axis, are adequate to separate the euthyroid cases from the other groups.

However, the diagnostic borderline values of $\mathbf{k}_{\mathbf{1}}$ deduced from the 24-hour uptake method, or from the bulk urine method (see Table III and Figure 1 ), do depend on the value selected for $k_{2}$; thus if a constant 24-hour uptake borderline is to be assumed, $k_{1}$ must increase if $k_{2}$ increases, and there is another different relation between $k_{1}$ and $k_{2}$ if a constant urinary parameter is assumed. Figure 1 illustrates these effects, showing curve 1 con- cave upwards and curve 2 downwards, and it is apparent that both curves cannot be correct over the full range of $k_{2}$ values, and one must choose some particular average value.

If cases with known cardiac or renal disease (10) are excluded, the following mean values of $\mathrm{k}_{\mathbf{2}}$ are to be considered:

$\begin{array}{lrrrrc}\text { Reference number } & 22 & 15,16 & 9 & 3 & \text { This paper } \\ \text { Number of cases } & 110 & 169 & 25 & 24 & 125 \\ \text { Mean value } 10^{3} \mathrm{k}_{2} & 2.59 & 2.6 & 2.02 & 1.84 & 1.51\end{array}$

A value of $k_{2}=2.2 \times 10^{-3}$ minute $^{-1}$ has therefore been taken as the weighted mean rate to be used in the conversion of parameters.

\section{Degree of agreement for boundary values of $k_{1}$}

On examination of Figure 4 it is evident that a horizontal borderline at $\mathrm{k}_{1}=3.9 \times 10^{-3}$ minute $^{-1}$ can be drawn to separate euthyroid and hyperthyroid cases, and another line at $k_{1}=0.23 \times 10^{-3}$ minute $^{-1}$ distinguishes (with some over-lapping) between euthyroid and hypothyroid cases. Figure 
TABLE VI

Comparison of diagnostic borderline values of $k_{1}$

\begin{tabular}{|c|c|c|c|}
\hline $\begin{array}{l}\text { Reference } \\
\text { number }\end{array}$ & $\begin{array}{l}\text { Method of } \\
\text { study }\end{array}$ & $\begin{array}{l}\text { Diagnostic } \\
\text { border }\end{array}$ & $\begin{array}{l}\text { Value of } \\
10^{2} k_{1} \text { in } \\
\text { minute }\end{array}$ \\
\hline $3,5,9$ & $\begin{array}{l}\text { Early clearance } \\
\text { rates }\end{array}$ & $\begin{array}{c}\text { Hyperthyroid/ } \\
\text { euthyroid }\end{array}$ & 3.7 \\
\hline 22 & Early uptake & $\begin{array}{l}\text { Hyperthyroid/ } \\
\text { euthyroid } \\
\text { Low/euthyroid }\end{array}$ & $\begin{array}{c}3.6-4.1 \\
0.30\end{array}$ \\
\hline $\begin{array}{l}1,2 \text { and } \\
\text { this paper }\end{array}$ & Early uptake & $\begin{array}{l}\text { Hyperthyroid/ } \\
\text { euthyroid } \\
\text { Low/euthyroid }\end{array}$ & $\begin{array}{l}3.9 \\
0.23\end{array}$ \\
\hline 4 & Urine & $\begin{array}{l}\text { Probably high/ } \\
\text { euthyroid }\end{array}$ & $4.2^{*}$ \\
\hline 15,16 & Urine & $\begin{array}{l}\text { Hyperthyroid/ } \\
\text { euthyroid }\end{array}$ & $3.7-4.2$ \\
\hline 13 & 24-Hour uptake & $\begin{array}{l}\text { Hyperthyroid/ } \\
\text { euthyroid }\end{array}$ & $3.0^{*}$ \\
\hline 20 & $\begin{array}{l}\text { Very early } \\
\text { uptake }\end{array}$ & $\begin{array}{l}\text { Hyperthyroid/ } \\
\text { euthyroid }\end{array}$ & $6.9^{*}$ \\
\hline 21 & Early uptake & $\begin{array}{l}\text { Hyperthyroid/ } \\
\text { euthyroid }\end{array}$ & $2.7^{*}$ \\
\hline
\end{tabular}

* For $\mathrm{k}_{2}=2.2 \times 10^{-8}$ minute $^{-1}$.

2 similarly gives a border somewhere between $\mathrm{k}_{\mathbf{1}}=3.6$ to $4.1 \times 10^{-3}$ minute $^{-1}$ for the data published by Berson, Yalow, Sorrentino, and Roswit (22). These results, and the others discussed earlier, are all compared in Table VI. For the hyperthyroid/euthyroid border the mean $k_{1}$ from the table is $4.0 \times 10^{-3}$ minute $^{-1}$, but for the various reasons given above it is felt that less weight should be given to the last three entries in the table. Moreover, the hypothyroid/euthyroid border of $0.30 \times 10^{-3}$ minute $^{-1}$ found from Figure 2 is based on only 5 low-uptakes cases, while the value of $0.23 \times 10^{-3}$ minute $^{-1}$ from Figure 4 is the result of 39 cases. Accordingly, the values finally selected for diagnosis are those given in Table VII, in which are included further limits suggested to distinguish cases of "very low" and "very high" thyroidal activity.

There is a good deal of experimental evidence supporting the value of $k_{1}=3.85 \times 10^{-3}$ minute $^{-1}$ adopted for the euthyroid/hyperthyroid diagnostic borderline, and it is felt that this value should be substantially correct.

For comparative purposes the corresponding limits for the clearance rate, the protein-bound iodine, and the 24-hour per cent uptake are also tabulated, but the latter values are not recommended for general application because of their dependence upon the renal rate factor $k_{2}$ and upon the type of collimator used with in vivo counting equipment. The limits tabulated for the proteinbound iodine test are those that give the best overall diagnostic agreement for the 174 cases tabulated in Table IV.

\section{Diagnostic results}

When the limits in Table VII are applied to the cases listed in Table IV and to a few others for which data have been accumulated from two or more independent tests, the percentage of apparently successful diagnostic results is 87 for the PBI determination, 94 for the $k_{1}$ test, and 88 for the 24-hour uptake tests when the latter is applied only to hypothyroid and euthyroid cases.

The few cases in which the $\mathrm{PBI}^{131}$ "conversion ratio" was tried were also nearly all euthyroid and low-uptake patients; the apparent success of

TABLE VII

Diagnostic borderline values finally adopted

\begin{tabular}{|c|c|c|c|c|}
\hline \multirow[b]{2}{*}{ Parameter } & \multicolumn{4}{|c|}{ Thyroid borderline status } \\
\hline & $\frac{\text { Very low }}{\text { Low }}$ & $\frac{\text { Low }}{\text { Euthyroid }}$ & $\frac{\text { Euthyroid }}{\text { High }}$ & $\frac{\text { High }}{\text { Very high }}$ \\
\hline $\begin{array}{l}\text { Uptake rate } 10^{2} \mathrm{k}_{1} \text { minute } \\
\text { Thyroidal plasma clearance rate* }\end{array}$ & 0.09 & 0.23 & 3.85 & 9.9 \\
\hline $\begin{array}{l}\text { 24-Hour uptake per cent } \dagger \\
\text { Protein-bound iodine\& }\end{array}$ & $\begin{array}{l}1.5 \\
3.9\end{array}$ & $\begin{array}{l}3.7 \\
8.0\end{array}$ & $\begin{array}{l}63 \\
62 \ddagger\end{array}$ & $\begin{array}{c}160 \\
82 \ddagger\end{array}$ \\
\hline $\begin{array}{l}\mu \mathrm{g} \text { per } 100 \mathrm{ml} \text {. } \\
\text { Combined PBI and } \mathrm{k}_{1}\end{array}$ & 2.8 & 3.9 & 8.2 & 10.0 \\
\hline$(\mathrm{PBI})^{1}+1.28 \log _{10}\left(10^{5} \mathrm{k}_{1}\right)$ & 2.90 & 3.72 & 6.17 & 7.00 \\
\hline
\end{tabular}

* For $\overline{\mathrm{V}}_{30}=16.3$ litre, see section 3 (a).

† Strictly for the collimator system described $(1,2)$; these values will not apply for open collimation.

$\ddagger$ Use of this parameter is not recommended for reasons given in section 3 (b).

For best diagnostic results for the cases tabulated in Table IV. 
this method (66 per cent) in this group was not very high, but of course better results might be obtained in sorting out hyperthyroid from euthyroid individuals.

Both the protein-bound iodine determination and the measurement of the rate of uptake of $\mathrm{I}^{181}$ are seen to give reasonably satisfactory results; in addition the 24-hour per cent uptake of $I^{181}$ is useful particularly for cases of low thyroidal activity where difficulty is experienced in making a complete collection of urine. As is well known, all of these tests can be spoilt by iodine or certain other types of medication, and great care is necessary to exclude this possibility. Finally, therapy with radioiodine may affect the subsequent tests in at least two ways : 1) There may be a residual amount of radioactivity in the thyroid gland, and this must be deducted from the observed amount taken up from the tracer dose; 2 ) in addition it may take a fairly long time for the stored protein-bound iodine to reach equilibrium at a new low-level value, even though the thyroidal uptake rate is lowered as the result of therapy.

\section{SUMMARY}

A series of 180 patients has been examined to determine their thyroidal status by the chemical protein-bound iodine and radioiodine methods, using the thyroidal uptake rate factor calculated from in vivo observations at 1,4 , or 24 hours after administration of the tracer dose. Good agreement has been found between the two methods in most cases.

The experimental results, and those of others using some other radioiodine methods, are used to derive suggested diagnostic borderline values of several parameters in common use.

\section{ACKNOWLEDGMENTS}

The authors are very grateful to Mr. C. Fitzgibbon who made the chemical determinations of protein-bound iodine, and to Mrs. M. Bates who carried out the measurements with radioiodine.

\section{REFERENCES}

1. Oddie, T. H., Meschan, I., and Wortham, J., Thyroid function assay with radioiodine. I. Physical basis of study of early phase of iodine metabolism and iodine uptake. J. Clin. Invest., 1955, 34, 95.
2. Oddie, T. H., Meschan, I., and Wortham, J., Thyroid function assay with radioiodine. II. Routine calculation of thyroidal and renal rate factors. J. Clin. Invest., 1955, 34, 106.

3. Myant, N. B., Corbett, B. D., Honour, A. J., and Pochin, E. E., Distribution of radioiodide in man. Clin. Sc., 1950, 9, 405.

4. Fraser, R., Hobson, Q. J. G., Arnott, D. G., and Emery, E. W., The urinary excretion of radioiodine as a clinical test of thyroid function. Quart. J. Med., 1953, 22, 99.

5. Ansell, G., Macgregor, A. G., Miller, H., and Wayne, E. J., The value of estimations of plasma protein-bound radioactive iodine as an index of thyrotoxicosis. "Radioisotopes Techniques," London, Her Majesty's Stationery Office, 1953; vol. I, p. 52.

6. Barry, M. C., and Pugh, A. E., Serum concentrations of radioiodine in diagnostic tracer studies. J. Clin. Endocrinol. \& Metab., 1953, 13, 980.

7. Stanley, M. M., and Astwood, E. B., Determination of the relative activities of antithyroid compounds in man using radioactive iodine. Endocrinol., 1947, 41, 66.

8. Berkson, J., Keating, F. R., Jr., Power, M. H., and McConahey, W. M., Determination of renal clearance of radioiodine. J. Applied Physiol., 1950, 2, 522.

9. McConahey, W. M., Keating, F. R., Jr., and Power, M. H., An estimation of the renal and extrarenal clearance of radioiodide in man. J. Clin. Invest., 1951, 30, 778.

10. Perry, W. F., and Hughes, J. F. S., The urinary excretion and thyroid uptake of iodine in renal disease. J. Clin. Invest., 1952, 31, 457.

11. Jaffe, H. L., and Ottoman, R. E., Evaluation of radioiodine test for thyroid function. J. A. M. A., 1950, 143, 515.

12. Werner, S. C., Quimby, E. H., and Schmidt, C., The use of tracer doses of radioactive iodine, $I^{m}$, in the study of normal and disordered thyroid function in man. J. Clin. Endocrinol., 1949, 9, 342.

13. Werner, S. C., Diagnostic technics with radioiodine. Med. Annals Dist. Columbia, 1953, 22, 12.

14. Fields, T., and LeRoy, G. V., An accurate method for the measurement of radioiodine in the thyroid gland by an external counter. Radiol., 1952, 58, 57.

15. Keating, F. R., Jr., Power, M. H., Berkson, J., and Haines, S. F., The urinary excretion of radioiodine in various thyroid states. J. Clin. Invest., 1947, 26, 1138.

16. Kelsey, M. P., Haines, S. F., and Keating, F. R., Radioiodine in the study and treatment of thyroid disease: a review. J. Clin. Endocrinol., 1949, 9, 171.

17. Reiss, M., Haigh, C. P., Hemphill, R. E., Maggs, R., Reiss, J. M., and Smith, S., Studies of the human 
thyroid function, measured by radio-iodine, and its relation to the basal metabolic rate. J. Endocrinol., 1952, 8, 1.

18. Lowrey, G. H., Beierwalters, W. H., Lampe, I., and Gomberg, H. J., Radioiodine uptake curve in humans. II. Studies in children. Pediatrics, 1949, 4, 627.

19. Crispell, K. R., Parson, W., and Sprinkle, P., A simplified technique for the diagnosis of hyperthyroidism, utilizing the one-hour uptake of orally administered Im. J. Clin. Endocrinol. \& Metab., 1953, 13, 221.

20. Clarke, K. H., and Aujard, R. L., Thyroid symposium III. The use of radioactive iodine $I^{110}$ in the diagnosis of hyperthyroidism: physical aspects. M. J. Australia, 1954, 1, 891.

21. Kriss, J. P., Uptake of radioactive iodine after intravenous administration of tracer doses. J. Clin. Endocrinol., 1951, 11, 289.

22. Berson, S. A., Yalow, R. S., Sorrentino, J., and Roswit, B., The determination of thyroidal and renal plasma $I^{m}$ clearance rates as a routine diagnostic test of thyroid dysfunction. J. Clin. Invest., 1952, 31, 141.

23. Silver, S., Fieber, M. H., and Yohalem, S. B., Blood levels after tracer doses of radioactive iodine in the diagnosis of thyroid disorders. Am. J. Med., 1952, 13, 725. 